\title{
MODELLING THE ELECTRICAL \\ PROPERTIES OF BLADDER TISSUE - \\ QUANTIFYING IMPEDANCE CHANGES DUE \\ TO INFLAMMATION AND OEDEMA
}

\author{
D. C. Walker ${ }^{1}$, R. H. Smallwood ${ }^{1}$, A. Keshtar ${ }^{2}$, B. A.Wilkinson ${ }^{3}$, F. C. Hamdy ${ }^{3}$, J. A. Lee ${ }^{4}$ \\ 1. Department of Computer Science University of Sheffield, Regent Court, 211 Portobello Street, \\ Sheffield, S1 4DP, United Kingdom \\ 2. Department of Medical Physics and Clinical Engineering, University of Sheffield, Floor I, Royal \\ Hallamshire Hospital, Glossop Road, Sheffield, S10 2JF, United Kingdom. \\ 3. Department of Urology, University of Sheffield, Floor I, Royal Hallamshire Hospital, Glossop \\ Road, Sheffield, S10 2JF, United Kingdom \\ 4. Department of Histopathology, Rotherham General Hospital, Moorgate Road, Rotherham, S60 \\ 2UD, UK \\ e-mail d.c.walker@sheffield.ac.uk
}

Short title: Modelling the electrical properties of bladder tissue

Classification numbers: $87.18 \mathrm{Bb}, 02.70 \mathrm{Dh}, 87.19 \mathrm{Xx}, 87.57 \mathrm{Ra}, 87.63 \mathrm{Pn}$

\begin{abstract}
Electrical Impedance Spectroscopy has been developed as a potential method for the diagnosis of carcinoma in epithelial tissues. An understanding of the influence of structural changes in the tissue on the properties measured using this technique is essential for interpreting measured data and optimisation of probe design. In contrast to other tissue types, carcinoma in Situ of the bladder gives rise to an increase in electrical impedance over the $\mathrm{kHz}-\mathrm{MHz}$ frequency range in comparison to normal tissue. Finite element models of the
\end{abstract}

\footnotetext{
- A Keshtkar,is now at: Medical Physics Department, Faculty of Medicine, Tabriz University of Medical Sciences,Tabriz-Iran
} 
urothelium and the underlying superficial lamina propria have been constructed and solved in order to ascertain the influence of structural changes associated with malignancy, oedema and inflammation on the measured electrical properties of the tissue. Sensitivity analysis of results from a composite tissue model suggests that the increase in lymphocyte density in the lamina propria associated with an inflammatory response to the infiltration of urine into the tissue may explain these unusual electrical properties.

\section{INTRODUCTION}

Electrical Impedance Spectroscopy, or EIS, is a non-invasive technique that has recently been developed for the diagnosis of pre-malignant changes in various epithelial tissues, including the squamous epithelium of the cervix, known as Cervical Intraepithelial Neoplasia, or CIN, (Brown et al. 2000), changes associated with Barrett's Oesophagus (Gonzalez-Correa et al. 1999), and more recently assessed for its ability to detect carcinoma in situ (CIS) in the bladder (Smallwood R H et al. 2002), (Wilkinson B A et al. 2002). The technique uses an alternating electrical current in the $\mathrm{kHz}-\mathrm{MHz}$ range, which is driven between two adjacent electrodes mounted on the tip of a pencil or endoscopic probe. The electrical potential is recorded between a second electrode pair and the transfer impedance is calculated, giving rise to a characteristic impedance spectrum for that particular tissue structure.

The electrical properties of tissues in the radio-frequency range are determined by the electrical properties of cellular components and the dimensions, internal structure and arrangements of the constituent cells. Hence tissues with different cellular structures, including those associated with various tissue pathologies, will give rise to characteristic impedance spectra. In the case of precancerous changes in squamous or transitional epithelia, structural changes include loss of the layer of flattened cells close to the surface and an increase in the volume of extra-cellular space (Feldman et al. 1984), (White et al. 1984).

Analysis of electrical impedance data measured from the cervix has suggested that the progression of CIN is characterized by a reduction in the low frequency impedance, $R$, and an increase in the characteristic frequency, $f_{c}$ (Brown et al. 2000), as shown in Figure 1a. The 
overall tendency for the electrical resistance to decrease with malignancy has been reported for several other tissues (Foster 1989), and in particular, a reduction in the low frequency impedivity $R$, has been measured using EIS for dysplasia in the oesophagus.

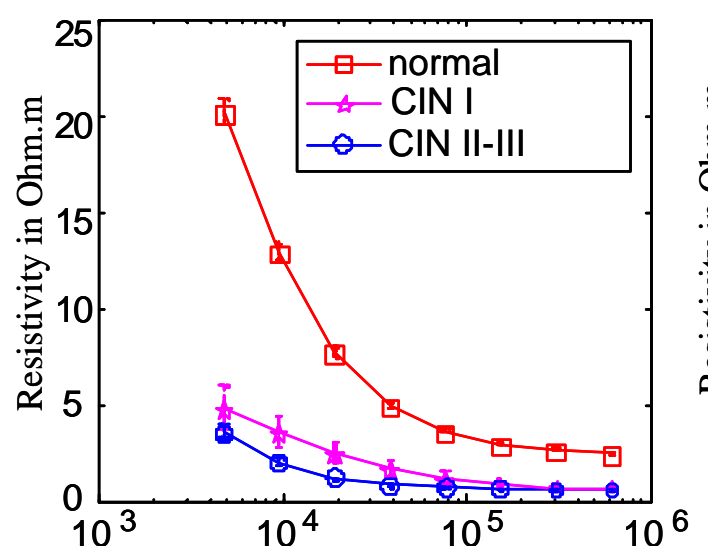

(a)

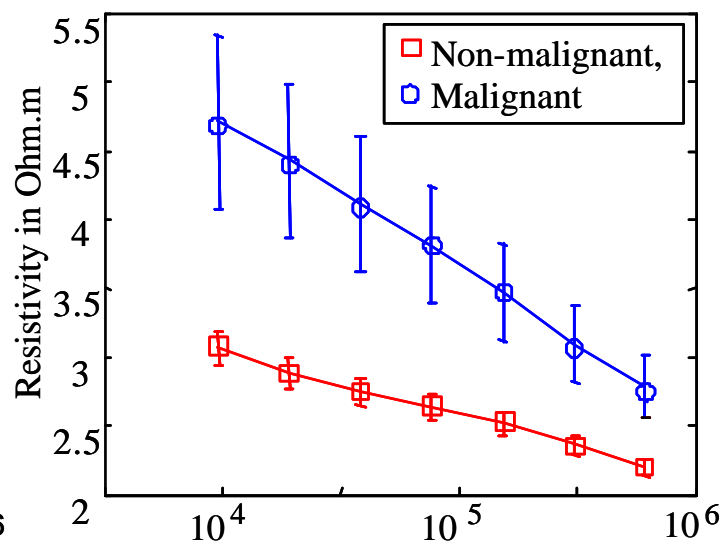

(b)

Frequency in $\mathrm{Hz}$

Figure 1

Measured transfer impedance spectra for a) cervix and b) bladder. Error bars in a) represent $\mathbf{9 5 \%}$ percentile range and $b) 1$ standard error of the mean

Modelling of the cervical epithelial changes associated with CIN has suggested that the reduction in the low frequency electrical impedance associated with precancerous development in the cervix can be attributed to the loss of cellular cohesion, or increase in extracellular volume (Walker 2001). In normal epithelial tissue, tight junctions at the tissue surface effectively confine low frequency current to a thin layer of surface mucus, whereas at higher frequencies, current penetrates the tissue surface to flow throughout the epithelium and the underlying, relatively high conductivity connective tissue, or stroma, thus giving rise to the characteristic dispersion. The lack of tight junctions, and substantially enlarged extracellular spaces (ecs) associated with CIN allow penetration of current across the whole frequency range, and hence a much smaller dispersion is observed. The magnitude and shape of the impedance response predicted by the model agree well with measurements made on cervical tissue in vivo (Walker D C et al. 2002). However, as illustrated in figure $1 \mathrm{~b}$, the opposite trend has been reported in the case of CIS in the bladder, where measurements on both in vivo and freshly excised tissue has revealed an increase in impedance across the frequency range for CIS, relative to normal tissue (Smallwood R H et al. 2002), (Wilkinson B A et al. 2002). It has been postulated that this unusual behaviour 
may be related to the effects of tissue oedema and inflammation that accompany malignant changes in bladder tissue. The study presented here attempts to test this hypothesis using finite element modelling to predict the electrical properties associated with inflammatory changes in tissue structure.

\section{METHODS}

\subsection{Finite Element Modelling of the electrical properties of tissue}

Computational modelling is a tool of growing importance in the fields of biology and medicine, providing a means of interrogating and increasing the understanding of the nonintuitive behaviour of complex systems across length scales that may span several orders of magnitude In the case of electrical problems, Finite Element (FE) modelling can be used to calculate an approximate solution for the potential distribution within a volume partitioned into a mesh of elements, which are connected at points termed nodes. If the electrical properties of the various materials in our cell or tissue model are known (or assumed), the resulting potential at any node on the model boundary, and hence the transfer impedance, can be calculated for any given set of applied currents. A full description of the theory of this technique and examples of applications to electrical biomedical problems can be found in (Miller C E et al. 1990) .In relation to the EIS technique, FE has been employed in order to examine the distribution of current flow in modelled epithelial tissue in order to optimise electrode array design (Jones D M et al. 2003), and to interpret measured impedance spectra in terms of tissue structure (Walker D C et al. 2002).

The level of detail contained in an FE model mesh is directly related to the total node number, and hence the computational resources required to solve the model. In order to include the effects of sub-cellular structure on the macroscopic measured electrical properties, a hierarchical method of modelling can be used. For instance, he lower, or cellular level hierarchy could consist of meshes of cells with dimensions typical of four different depths in normal and pre-cancerous cervical epithelium, as determined from a study of the histology of normal and pre-cancerous epithelium (Walker D C et al. 2003). These 
meshes can then be used to calculate the anisotropic electrical properties parallel and perpendicular to the cell axes, which are then assigned as material properties in the second hierarchy model. In the case of $\{$ Walker D C, $2002 \# 69\}$, this consists of four epithelial layers representing superficial, intermediate, parabasal and basal cell types, a layer representing the underlying connective tissue, and a surface layer of variable thickness, representing a thin layer of cervical mucus. In order to obtain a value for the properties of the connective tissue, or stroma, for assignment in this second level model, the conductivity of unfixed, freshly excised healthy cervical stroma obtained, with ethical committee approval and consent, from hysterectomy patients was measured. The mucus layer was assigned similar properties to those used for extra-cellular fluid. A typical cellular-level mesh, and the macroscopic model are shown in figure 2. A more detailed description of this hierarchical modelling process, including the cell dimensions and electrical properties used are given in (Walker D C et al. 2002) and (Walker 2001).

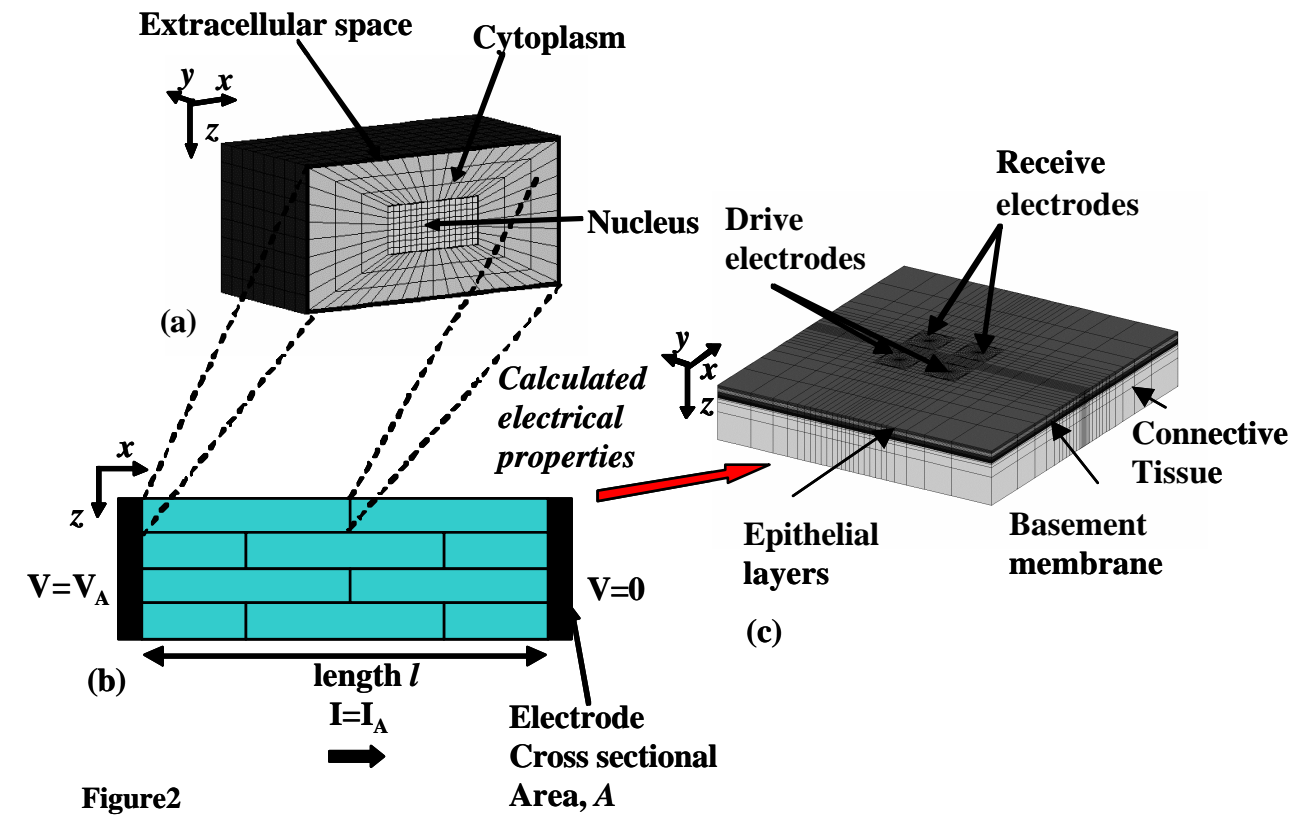

Schematic of hierarchical modelling process. Single cell meshes (a) of various sizes are used to calculate the effective complex electrical properties of different epithelial layers (b). These effective conductivities are then specified as epithelial layer material properties in the macroscopic model (c).

\subsection{Modelling changes in the lamina propria}

The decision to model cervical stroma as an electrically isotropic layer was justified as the changes associated with CIN, by definition, are confined to the epithelium. By contrast, the connective tissue, or lamina propria of the bladder can undergo changes early on in the 
development of CIS. The purpose of the epithelial lining of the bladder, or urothelium, is to provide a physical protective barrier between the urine and the lamina propria. Once this barrier function is compromised due to the loss of tight junctions, superficial umbrella cells and widening extracellular space, urine is able to penetrate into the lamina propria thus inducing inflammatory changes. Oedema, or an increase in extracellular fluid volume due to capillary leakage, is also commonly associated with early changes in the bladder. As the urothelium is significantly thinner than the epithelium of the cervix (approximately $60 \mu \mathrm{m}$ compared with $300 \mu \mathrm{m}$ ), the contribution of changes in the connective tissue in the bladder would be expected be more significant than the contribution of the cervical stroma to the measured electrical properties.

Figure 3 shows typical histology sections obtained from bladder diagnosed as normal and exhibiting symptoms of CIS. Features typical of oedema and inflammation, including the increased lymphocyte density and dilation of capillaries are visible in the lamina propria of the CIS tissue. These changes will also be accompanied by an increase in capillary wall permeability and hence an increase in fluid in the surrounding connective tissue.

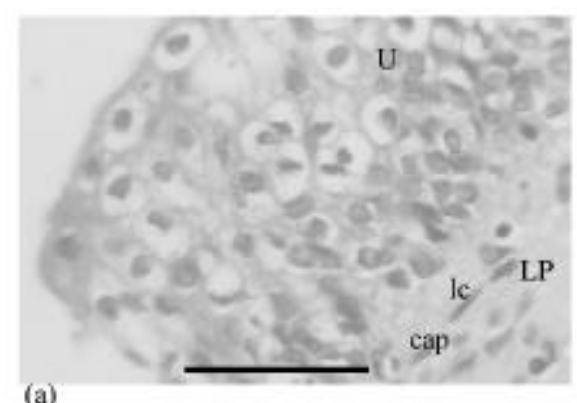

(a)

Typical histology sections derived from (a) normal and (b) CIS bladder, showing urothelium, superficial lamina propria. $U=$ urothelium, $L P=$ lamina propria, cap=capillary and $l c=$ lymphocyte.

In order to obtain estimates of the changes in capillary size and vessel and lymphocyte density associated with normal and pathological bladder tissue, an analysis of a number of digital images of histological slides similar to those shown in Figure 3 was undertaken. Sections were obtained from biopsies classified by a bladder pathologist as either normal, CIS or Ca (invasive carcinoma), with additional gradings according to overall intactness of the urothelium, and degree of inflammation and oedema. This small study suggested that the 
changes in capillary density and size were relatively insignificant, but the increase in lymphocyte population was marked. The sensitivity of the electrical properties of bladder tissue due to changes in lymphocyte density and capillary leakiness, with negligible change in diameter or density, was then assessed using hierarchical FE models.

\subsubsection{Modelling Capillary Leakiness}

The primary purpose of the capillary network is to regulate the exchange of chemical products and ymphocytes between the blood and surrounding tissue. Unlike larger blood vessels such as arteries and veins, capillary walls do not contain smooth muscle, but consist of a single layer of endothelial cells, connected by tight junctions, and surrounded by a thin basement membrane. The paracellular permeability of the capillary wall is modulated by widening of the gaps between adjacent endothelial cells. Widening of these gaps results in the leakage of fluid into the surrounding tissue, resulting in tissue swelling, or oedema. Measurements of electron micrographs of normal capillary walls revealed these gaps to be approximately $0.02 \mu \mathrm{m}$ in width, with an oblique overlap of approximately $1 \mu \mathrm{m}$ between adjacent endothelial cells. Studies of rat lung endothelium revealed that gaps widened up to $0.5 \mu \mathrm{m}$ in the presence of inflammatory agents (Baluk P et al. 1997).

Although it is relatively straightforward to assemble FE model meshes that represent vessels as tube-like structures, inclusion of cellular level detail in the walls of these meshes would be significantly more complicated. For this reason, another model hierarchy was introduced in order to capture the electrical properties of vessel walls with varying separation between adjacent endothelial cells. Figure 4a shows the geometry of the model mesh used to do this. Modelled endothelial cells consisted of cytoplasm and an 8nm thick cytoplasmic membrane. Cells were $12 \mu \mathrm{m}$ in length and $0.5 \mu \mathrm{m}$ in height and overlapped by $1 \mu \mathrm{m}$. The oblique separation between the cell edges was varied between $0.02 \mu \mathrm{m}$ and $0.5 \mu \mathrm{m}$ in five steps. A $0.1 \mu \mathrm{m}$ continuous layer representing basement membrane was included representing the outer layer of the capillary wall. The electrical conductivity and permittivity values assigned to the model cytoplasm and membrane are given in Table 1 . Basement membrane was assigned the published electrical properties of tendon (Gabriel et al. 1996). 


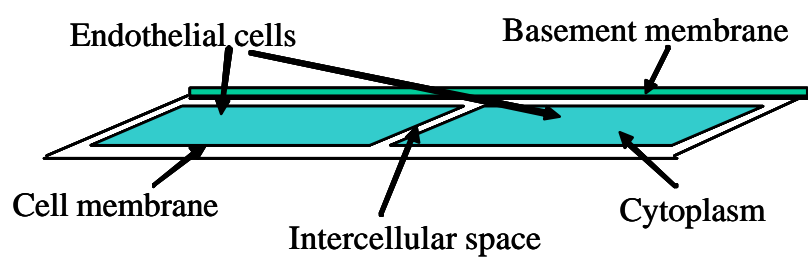

(a)

Figure 4

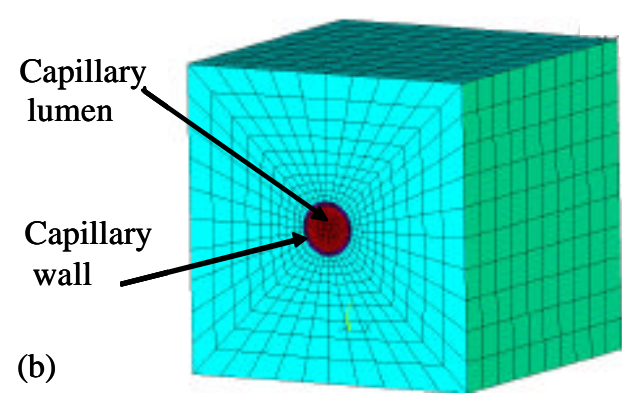

(a) Schematic of capillary wall model (b) Finite element mesh of $6 \mu \mathrm{m}$ diameter capillary in homogenous tissue block

Currents in the frequency range $100 \mathrm{~Hz}-10 \mathrm{MHz}$ were applied at the boundaries of the model in the $\mathrm{x}$ and $\mathrm{z}$ planes, and the FE equations were assembled and solved to calculate the resulting potentials at these planes, as shown for bricked epithelial cells in Figure $2 b$. The effective electrical transfer impedance $Z_{T}$ and hence the effective space averaged complex electrical conductivities, $\sigma e^{*}$ parallel and perpendicular to the endothelial cell orientation were calculated using:

$$
Z_{T}=\frac{V}{I}
$$

and:

$$
\sigma_{e}^{*}=\frac{l}{Z A}
$$

where $I$ is the applied boundary current, $V$ is the calculated boundary potential, $A$ is the cross-sectional area transverse to the direction of current flow, and $l$ is the distance between the two boundary planes under consideration. The effective conductivity values calculated at each frequency were then assigned as vessel wall element properties in the second hierarchy model, shown in Figure 4b. This mesh consists of a tube of $6 \mu \mathrm{m}$ diameter and $0.6 \mu \mathrm{m}$ wall thickness in a $34 \mu \mathrm{m} \times 34 \mu \mathrm{m} \times 34 \mu \mathrm{m}$ block of homogenous material, representing the wall thickness, and capillary size and density estimated from both normal and inflamed/malignant tissue sections as outlined above.

In order to include the effect of increased fluid content in the surrounding tissue, the conductivity assigned to the ecs in this second level model was varied between 1.1 and $1.9 \mathrm{~S} / \mathrm{m}$, with the lowest conductivity associated with the smallest endothelial cell separation, and so on. This factor of variation was based on the supposition that tissue with moderate to 
severe oedema will approximately double its normal fluid content. The interior of the modelled vessel was assigned an electrical conductivity of $1.5 \mathrm{~S} / \mathrm{m}$ (the published value for human plasma at body temperature) (Geddes et al. 1967)and the same permittivity as ecs.

\subsubsection{Modelling varying lymphocyte density $(\mathrm{Ld})$}

The methodology of mode lling lymphocytes was based on that developed previously for modelling closely packed cells in the epithelial layers. Model meshes consisted of cuboidal cells comprising cytoplasmic membrane, cytoplasm, nuclear membrane and nucleus, surrounded by an outer envelope of ecs, as shown in figure 2a. Modelled lymphocytes were $12 \mu \mathrm{m}$ in length and nuclear:cytoplamic ratio was 0.8 . Lymphocyte density was varied between $10 \%$ and $80 \%$ of total volume by altering the volume of ecs surrounding the cell. Five models were solved, representing lymphocyte volume densities of $10 \%, 20 \%, 40 \%$, $60 \%$ and $80 \%$. Electrical properties for the various model compartments are given in Table 1. No oedema was considered in this case, so ecs was assigned a conductivity of $1.1 \mathrm{~S} / \mathrm{m}$. Boundary conditions were applied to the model as described above, in order to calc ulate the effective space averaged complex electrical conductivities, $\sigma e^{*}$, as shown in Figure $2 \mathrm{~b}$. human plasma at body temperature) (Geddes et al. 1967)and the same permittivity as ecs.

\begin{tabular}{|l|l|l|l|}
\hline $\begin{array}{l}\text { Cellular } \\
\text { compartment }\end{array}$ & $\begin{array}{l}\text { Conductivity, } \sigma \\
(\mathbf{S} / \mathbf{m})\end{array}$ & $\begin{array}{l}\text { Relative } \\
\text { Permittivity, } \varepsilon_{\mathbf{r}}\end{array}$ & $\begin{array}{l}\text { Capacitance } \\
\mu \mathbf{F} / \mathbf{m}\end{array}$ \\
\hline $\begin{array}{l}\text { Extracellular } \\
\text { space }\end{array}$ & 1.1 & 72 & \\
\hline Cell membrane & $1 \times 10^{-1}$ & & 0.01 \\
\hline Cytoplasm & 0.6 & 86 & 0.007 \\
\hline $\begin{array}{l}\text { Nuclear } \\
\text { membrane }\end{array}$ & $2 \times 10^{-3}$ & & \\
\hline Nuclear interior & 0.8 & 145 & \\
\hline
\end{tabular}

Table 1 Electrical properties used to model cellular compartments, derived from average values published in literature. Cell membrane thickness $=8 \mathrm{~nm}$, nuclear membrane thickness $=40 \mathrm{~nm}$

\subsubsection{Modelling the macroscopic level electrical properties}

In order to generate model impedance spectra that can be compared to measured data, or analysed for sensitivity to particular parameters,, a single macroscopic tissue level mesh was used, similar to that shown in Figure 2c. The model is meshed in such a way that the surface distribution of nodes directly mimics the configuration of the current electrodes in the impedance measurement system, i.e. four circular electrodes of diameter $0.5 \mu \mathrm{m}$, and edge 
separation $0.2 \mu \mathrm{m}$. Current boundary conditions can then be applied to nodal sets representing the drive electrodes, and constraints which force zero potential gradient to nodal sets representing the measurement electrodes This ensures that a single voltage value is calculated at these nodes, and allows an effective transfer impedance to be calculated using equation 2.1, where in this case, $I$ is the current applied at the drive electrodes, and $V$ is the single value of potential calculated at the measurement electrode.

Model results were 'calibrated' in order to obtain size and electrode independent values of transfer impedance or impedivity. This was done by solving a model using the standard macroscopic model mesh and boundary conditions, but fixing the conductivity of every element in the model to a value of $1 \mathrm{~S} / \mathrm{m}$. The transfer impedance calculated using this model yielded a calibration constant for all other model solutions. It was then possible to directly compare the calibrated impedances derived from the tissue model, with those obtained from in vivo measurements.

\subsection{Sensitivity analysis of macroscopic effects of epithelial anisotropy, oedema and inflammation}

The purpose of sensitivity analysis is to determine the dependence of one or more outcomes on one or more influencing parameters. When there are more than two influencing parameters to be considered, this is known as multivariate sensitivity analysis. The statistical method employed for sensitivity analysis is multi-linear regression. This technique evaluates the degree of linear response between each output parameter, $Y$, and each input parameter $X i$. In effect, the technique calculates the best-fit parameters to the multidimensional linear equation:

$$
Y=a+b_{1} X_{1}+b_{2} X_{2}+b_{3} X_{3}+\ldots
$$

In terms of the finite element modelling, the full set of dependent outcomes $(Y \mathrm{~s})$ can be considered as the complex electrical impedances at each of the frequencies considered, and the influencing parameters $(X i s)$ are our model inputs, which include both geometric and electrical parameters at each model hierarchy. A full sensitivity analysis, including all input and output parameters, is not tractable in terms of computation time required. However, a limited analysis can be used in order to assess the relative effects of oedema, inflammation in 
the lamina propria and changes in anisotropy associated with malignant transformation in the urothelium on the measured impedance spectrum.

\subsubsection{Input parameters}

Previous studies have demonstrated that malignant changes in the cervical epithelium, including loss of differentiation and an increase in ecs have large affects on the measured impedance spectrum. The object of this study was to quantitatively compare similar changes in the urothelium with those associated with oedema and inflammation, which also affect the underlying connective tissue layer. In order to do this, a total of 45 models were solved at 11 different current frequencies by combining the sets of previously calculated epithelial/lamina propria electrical properties as follows:

1. three ets of epithelial layer properties calculated as shown in figure $2 \mathrm{a}-\mathrm{b}$ using different cell morphologies to represent progressive loss of differentiation

2. three sets of epithelial layer properties calculated using cell models with increasing extracellular volume distribution (figure 2a-b), combined with lamina propria properties derived from models with increasing capillary leakiness and lamina propria ecs conductivity (figure $4 \mathrm{~b}$ ) to simulate increasing levels of oedema.

3. Five sets of lamina propria properties corresponding to increasing lymphocyte density (see section 2.2.2 and figure $2 \mathrm{a}$ and $\mathrm{b}$ ), to simulate increasing levels of inflammation.

\section{$\underline{\text { Loss of differentiation }}$}

Typical cell sizes in different layers of cervical epithelium have previously been obtained from a literature and histology based study (Walker D C et al. 2003), but due to the lack of availability of good quality images, it was not possible to carry out a similar study for urothelium. For this reason, epithelial models were solved for three different sets of superficial layer cell shapes which approximately represent stretched normal urothelium, relaxed normal urothelium and urothelium exhibiting CIS. These cell models were in turn combined with different widths of extracellular space (ecs) distributions, corresponding to those used to model normal and CIN3 cervical tissue, as described in (Walker D C et al. 
2002), and an intermediate set of values. Previous modelling work has suggested that increases in nuclear:cytoplasmic ratio does not have a significant effect on the electrical properties of tissue (Walker 2001), so this parameter was not considered during this study. Actual cell sizes used are given in Table 2 and ecs in each layer in Table 3, giving a total of 9 different cell morphology/ecs width combinations.

\section{Oedema}

Oedema is defined as an increase in the extracellular fluid volume of a tissue. This condition was modelled by associating an increase in lamina propria conductivity with each increase in ecs width in the epithelium. Effective epithelial transfer properties calculated using the method illustrated in figure $2 \mathrm{a}-\mathrm{b}$ with ecs widths given in Table 3 were combined with the first, third and fifth sets of lamina propria properties shown in figure 5 (with the smallest ecs widths associated with the largest lamina propria impedance).

\begin{tabular}{|l|l|l|l|}
\hline Layer no. & \multicolumn{3}{|l|}{ Cell dimensions, $(\mathbf{x}$ y $\mathbf{z}$ in $\boldsymbol{\mu m})$} \\
\hline & Set 1 & Set 2 & Set 3 \\
\hline 1. Superficial & $60 \times 60 \times 6$ & $30 \times 30 \times 6$ & $18 \times 18 \times 12$ \\
\hline 2. Intermediate & $15 \times 15 \times 15$ & $15 \times 15 \times 15$ & $15 \times 15 \times 15$ \\
\hline 3. Basal & $12 \times 12 \times 12$ & $12 \times 12 \times 12$ & $12 \times 12 \times 12$ \\
\hline $\boldsymbol{A}_{\boldsymbol{i}}$ & 2.42 & 1.58 & 1.25 \\
\hline $\boldsymbol{A}_{\boldsymbol{i N}}$ & 1.38 & 0.90 & 0.71 \\
\hline
\end{tabular}

Table 1 Dimensions of epithelial cellular compartments used in sensitivity analysis, and corresponding anisotropy index $A_{i}$ and normalised anisotropy index $A_{i N}$ (see equation 2.4)

\begin{tabular}{|l|l|l|l|}
\hline Layer no. & \multicolumn{3}{|l|}{$\begin{array}{l}\text { Extracellular space width per cell, in } \\
\mu \mathbf{m}\end{array}$} \\
\hline & Set 1 & Set 2 & Set 3 \\
\hline 1. Superficial & 0.05 & 0.1 & 0.2 \\
\hline 2. Intermediate & 0.1 & 0.2 & 0.4 \\
\hline 3. Basal & 0.2 & 0.4 & 0.8 \\
\hline Mean value & 0.12 & 0.23 & 0.47 \\
\hline LP conductivity S/m & 1.10 & 1.45 & 1.92 \\
\hline $\mathbf{E}_{\mathbf{i N}}$ & 0.38 & 0.86 & 1.76 \\
\hline
\end{tabular}

Table 3 Dimensions of epithelial extracellular space distribution and lamina propria (LP) conductivity used to model oedema in sensitive ity analysis. $\mathrm{F}_{\mathrm{N}}=$ normalised oedema index (see text for details)

\section{$\underline{\text { Inflammation }}$}

Physiologically, this relates to the effect of urine penetrating wider ecs gaps, resulting in an increase invasion of inflammatory cells into the underlying connective tissue. At the microscopic level inflammation was modelled by firstly solving single cell models with different volumes of ecs, as described in section 2.2.2 above and illustrated in figure 2a-b. In 
order to vary both oedema and inflammation in the lamina propria, the conductivity of the ecs in the lymphocyte models was varied as described in Table 3, the resulting effective transfer impedance properties assigned as material properties in the layer labelled 'connective tissue' in figure $2 \mathrm{c}$. The lymphocyte volume densities used to provide lamina propria properties are given in Table 4.

\begin{tabular}{|l|l|l|l|l|l|}
\hline $\begin{array}{l}\text { LP lymphocyte } \\
\text { volume density }\end{array}$ & $\mathbf{1 0 \%}$ & $\mathbf{2 0 \%}$ & $\mathbf{4 0 \%}$ & $\mathbf{6 0 \%}$ & $\mathbf{8 0 \%}$ \\
\hline $\mathbf{I}_{\mathrm{iN}}$ & 0.24 & 0.48 & 0.95 & 1.43 & 1.90 \\
\hline
\end{tabular}

Table 4 Lymphocyte volume density in lamina propria (LP) used to model inflammation in sensitivity analysis. $I_{\mathrm{i}_{\mathrm{N}}}=$ normalised oedema index (see text for details)

\section{Normalisation of input parameters}

In order to carry out the multiple linear regression, it is necessary to normalise each of the input values tested. This is trivial in the case of the oedema and lymphocyte density, where each of the values is simply divided by the mean value for that parameter set, to provide an Oedema Index $(E i)$ and an Inflammation Index $(\mathrm{Ii})$. However, quantification of epithelial cell morphology is slightly more complex. For this reason, the concept of an Anisotropy Index is introduced. The calculation of this index is based on the observation that there is little change in the size of the cells in the basal layer with pathology. The cell lengths in the intermediate and superficial layers are then defined as a ratio of this size, and the sum of these calculated to give an index of anisotropy, Ai. This is normalized by dividing by three, so a perfectly isotropic tissue would have an $A i$ equal to 1:

$$
A_{i}=\frac{1}{3} \sum_{n=1}^{3}\left[\frac{\text { length in layer } \mathrm{n}}{\text { lengthin layer } 1}\right]
$$

Finally, each of the input parameters is normalized by dividing each parameter by the mean value of its set. This results in the final set of input parameter values:

$$
\begin{array}{ll}
A_{i N} & {[1.38,0.9,0.71]} \\
\mathrm{E}_{i N} & {[0.375,0.86,1.76]} \\
I_{i N} & {[0.24,0.48,0.95,1.43,1.90]}
\end{array}
$$

\subsubsection{Output parameters}

The FE model is set up in order to generate complex values of transfer impedivity at each of the 11 current frequencies considered, resulting in a total of 22 output values. In order to 
reduce the number of output parameters, modelled impedance spectra can be parameterised. The electrical behaviour of a single cell with membrane capacitance $C$, cytoplasmic resistance $S$, surrounded by extracellular space of resistance $R$ can be represented as the simple electrical circuit consisting of a resistor $R$, in parallel with a second resistor, $S$ and a capacitor $C$. Impedance curves measured from tissues can be characterized in terms of these values, known as the Cole parameters (Cole 1941) using:

$$
Z(f)=Z_{\infty}+\frac{Z_{0}-Z_{\infty}}{1+\left(j f / f_{c}\right)^{\alpha}}
$$

where $Z_{0}$ is the low frequency impedance, often assumed to be equivalent to the extracellular resistance, $R$ and $Z_{\infty}$ is the high frequency impedance, given by:

$$
Z_{\infty}=\frac{R S}{R+S}
$$

and $f_{c}$ is the characteristic frequency at the centre of the dispersion. This is usually defined as the frequency where the gradient of the curve, $d Z / d f$, is at its maximum value, and is related to the Cole parameters by:

$$
f_{c}=\frac{1}{2 \pi(R+S) C}
$$

This method of parameterising the spectra in terms of the Cole model is routinely used to analyse the data collected in vivo from various pathologies of cervical tissue (Brown et al. 2000).

\subsubsection{Multiple Linear Regression}

Cole parameters $R, f_{c}$ and $S$ were fitted to the calibrated modelled impedance spectra using custom-written code in Matlab. It was observed that $R$ and $f_{c}$ could easily be derived in all cases, and the new curves generated using these derived parameters fit very well with the original curves in the low and intermediate frequency ranges. However, derivation of the $S$ parameter was not as straightforward. One reason for this was that the modelled curves did not 'flatten out' above the characteristic frequency, but the impedivity continued to decrease, and in some cases a second dispersion was visible. This non-intuitive behaviour of the model has previously been observed (Walker 2001) and is explained by the fact that the current- 
depth distribution changes significantly with frequency, and each tissue layer has its own dispersive properties due to different cell sizes and densities. For this reason, the $S$ parameter was excluded from the sensitivity analysis on the grounds of being unreliable, and the real component of impedivity at the highest modelled frequency $(10 \mathrm{MHz}), \mathrm{Z}_{\max }$, was considered as the third output parameter.

Vectors corresponding to $R, f_{c}$ and $Z_{\max }$ were then assembled, corresponding to the $Y$ vector of outputs in the linear regression. For each entry in the vector, a matrix row was assembled, consisting of the normalised values of each of the model inputs used to generate each output parameter.

Multiple linear regression was then carried out for each output parameter in turn, using the Minitab package. Correlation coefficients between each set of input and output parameters were also calculated. A correlation coefficient of 1.0 represents a perfect linear relationship, which decreases as the relationship becomes less linear (though remains high for strong nonlinear relationships e.g. a quadratic dependence), and decreases to zero for the case when there is no relationship between inputs and outputs. A p-value is also quoted for each input in every regression test. This represents the statistical significance of testing the null hypothesis that the output is independent of the input i.e. represents the probability that a rejecting the null hypothesis would be a mistake. A small $\mathrm{p}$-value would then indicate that the output, $Y$, has a strong linear dependence on input $X i$. Finally, the $R_{s q}$ parameter for each egression analysis represents the percentage of the total variance in each output described by the relationships fitted in each regression analysis.

\section{RESULTS}

\subsection{Lamina propria (first level) results}

\subsubsection{Effect of Capillary Leakiness}

Figure 5 shows the real part of the effective complex transfer impedivity (or resistivity) calculated perpendicular to the orientation of the endothelial cells (i.e. across the capillary wall), as a function of the gap between the endothelial cells, and extra-vascular conductivity. 
These results suggest that, as expected, there is an inverse linear relationship between increased fluid content, with a doubling of fluid volume resulting in a factor of $\sim 2$ reduction in resistivity. The actual structure of the capillaries has little effect on the electrical properties of the lamina propria, with only a slight dispersion visible in the $\mathrm{MHz}$ region.

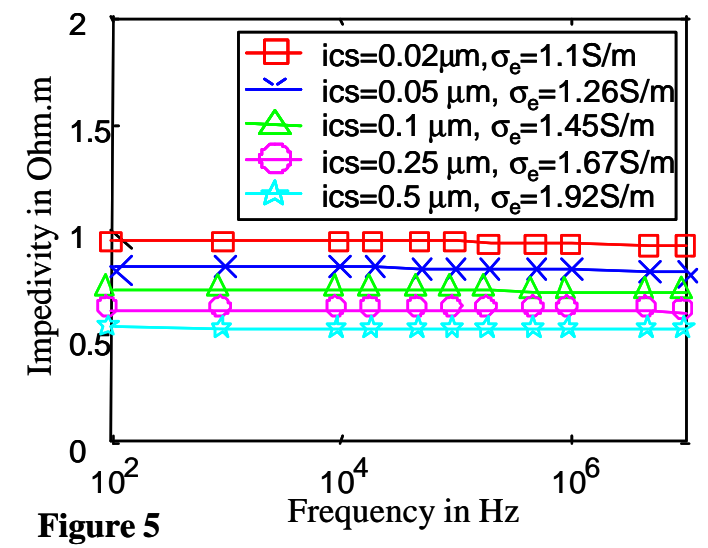

Modelled impedivity spectra obtained from capillary wall model with increasing endothelial intercellular space (ics) and extravascular conductivity on local tissue (superficial lamina propria) scale

\subsubsection{Effect of lymphocyte density}

The effect of increasing lymphocyte volume density (i.e. total tissue volume composed of lymphocytes as opposed to homogenous ecs) on the frequency dependent resistivity is shown in Figure 6. Inspection of this figure suggests that an increase in lymphocyte density would lead to an increase in electrical resistivity, across the frequency range of interest, with an increasing dispersion in the $10^{5}-10^{6} \mathrm{~Hz}$ range. These changes are of the order of those predicted for changes in epithelial cell morphology.

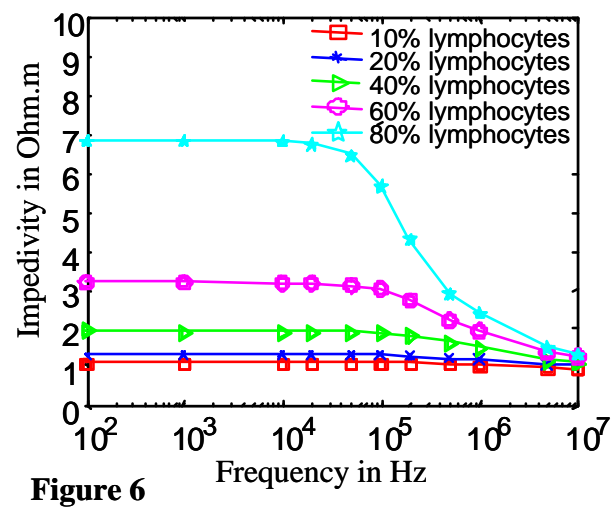

Modelled impedivity spectra obtained from tissue containing increasing volume density of lymphocytes (inflammation)3.2 Macroscopic (second level) impedance spectra and sensitivity analysis 


\subsubsection{Effect of loss of differentiation}

The modelled resistivity spectra obtained when epithelial anisotropy is reduced, for median levels of oedema and inflammation $\left(\mathrm{E}_{\mathrm{iN}}=0.86, \mathrm{I}_{\mathrm{iN}}=0.95\right)$ are shown in figure 7 . Low frequency impedivity is reduced, and characteristic frequency increased as $\mathrm{A}_{\mathrm{iN}}$ decreases.

The results of the sensitivity analysis are shown in Table 5. The parameters shown confirm that there is a direct correlation between epithelial anisotropy and low frequency impedance $R$, and an inverse correlation with characteristic frequency $f_{c}$ (i.e. $f_{c}$ increases as $A_{i N}$ decreases). The high frequency electrical properties are unaffected by changes in cell morphology.

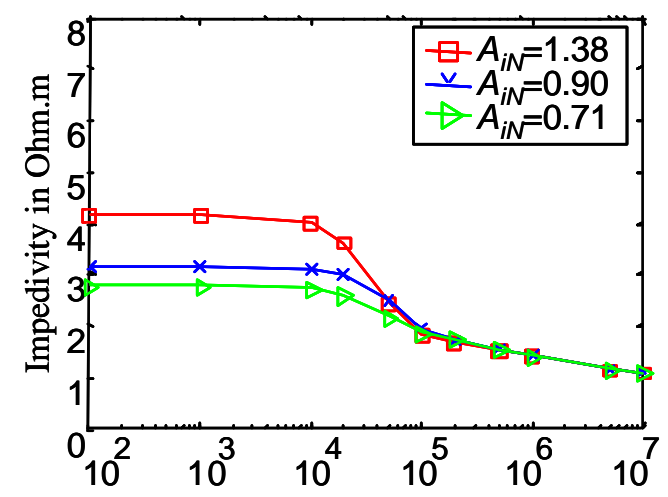

Figure 7

Effect of increasing epithelial anisotropy index, $A_{i N}$ on modelled macroscopic impedivity

\subsubsection{Effect of increasing oedema}

Figure 8 shows the predicted effect of increasing the epithelial extracellular space width and lamina propria conductivity, while cell morphology and lamina propria lymphocyte density remain constant at median values $\left(\mathrm{A}_{\mathrm{iN}}=0.90\right.$ and $\left.\mathrm{I}_{\mathrm{N}}=0.95\right)$. As expected, increasing the volume of ecs in the epithelium reduces the low frequency resistivity. The characteristic frequency is also increased with increasing oedema, while the high frequency resistivity is reduced.

The sensitivity analysis (Table 5) shows a strong inverse correlation between oedema and $R$, a weaker but nonetheless significant inverse correlation between oedema and $Z_{\max }$, and direct correlation between $E_{i N}$ and $f_{c}$. 


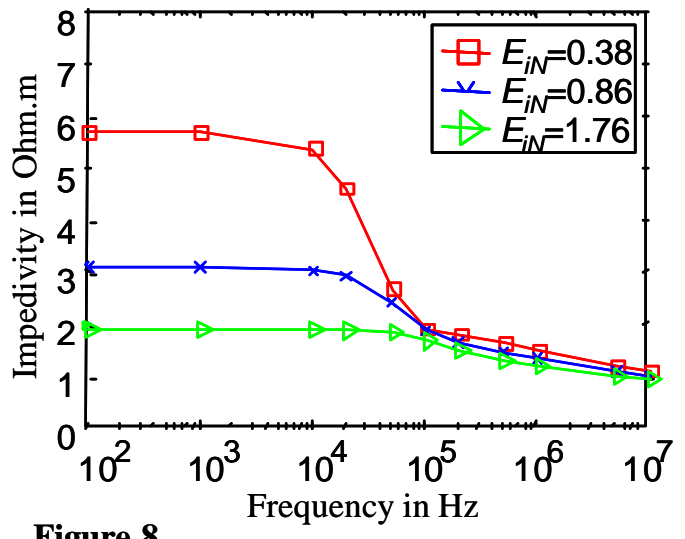

Figure 8

Effect of increasing oedema $E_{i N}$ on modelled macroscopic impedivity

\subsubsection{Effect of Inflammation}

Figure 9 suggests that an increase in the lymphocyte population would increase $R, f_{c}$ and $Z_{\max }$. Inspection of Table 5 suggests that the influence of lymphocyte density on $Z_{\operatorname{maxf}}$ is greater than that of the other two input parameters considered.

\begin{tabular}{|c|c|c|c|c|c|c|}
\hline \multirow{2}{*}{$\begin{array}{l}\text { Output } \\
\text { params. }\end{array}$} & & & \multicolumn{3}{|c|}{ Input variables } & \multirow[t]{2}{*}{$\mathbf{R}_{\mathrm{sq}}$} \\
\hline & & & $\overline{A_{i}}$ & $\overline{E_{i}}$ & $\boldsymbol{I}_{i}$ & \\
\hline \multirow[t]{4}{*}{$\boldsymbol{R}$} & $\mathbf{a}$ & 2.5 & & & & \\
\hline & b & & 2.41 & -2.91 & 2.65 & \\
\hline & p & & 0.000 & 0.000 & 0.000 & $84.7 \%$ \\
\hline & c.c. & & 0.26 & -0.64 & 0.62 & \\
\hline \multirow[t]{4}{*}{$\log _{10}\left(f_{c}\right)$} & $\mathbf{a}$ & 4.5 & & & & \\
\hline & $\mathbf{b}$ & & -0.407 & 0.548 & 0.331 & \\
\hline & p & & 0.000 & 0.000 & 0.000 & $90.4 \%$ \\
\hline & c.c. & & -0.28 & 0.77 & 0.49 & \\
\hline \multirow[t]{4}{*}{$Z_{f}$} & $\mathbf{a}$ & 0.94 & & & & \\
\hline & $\mathbf{b}$ & & 0.000 & -0.115 & 0.260 & \\
\hline & p & & 1.000 & 0.000 & 0.000 & $98.1 \%$ \\
\hline & c.c. & & -0.000 & -0.38 & 0.92 & \\
\hline
\end{tabular}

Table 5 Results of multiple linear regression and correlation testing on macroscopic level model spectra a and $b$ are coefficients of sensitivity analysis, $p$ indicates signficance values and c.c. correlation coefficients

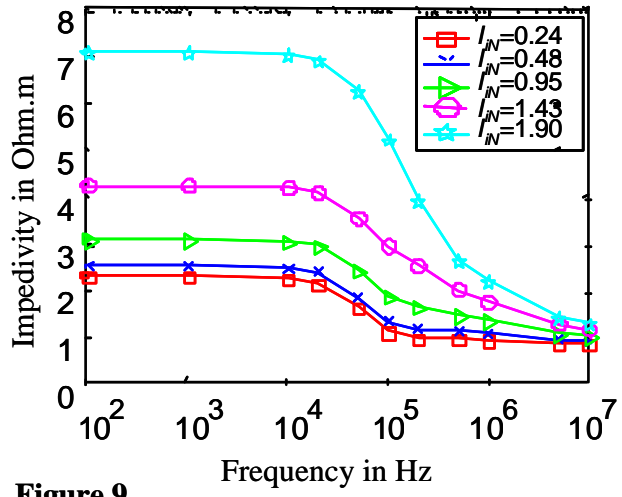

Figure 9

Effect of increasing inflammation $I_{i N}$ on modelled macroscopic impedivity 


\subsection{Comparison with measured data}

Results from impedance measurements on freshly excised bladder tissue have been published elsewhere (Smallwood R H et al. 2002), (Wilkinson B A et al. 2002), but are included here for the purpose of comparison with the modelling results. Following the impedance measurements, the biopsies from the site of measurement were examined by a bladder pathologist and assigned indices ranging from 0-4 according to levels of oedema and inflammation in the lamina propria, and an additional index relating to the condition of the urothelium.

Figure 10a shows the resistivity calculated using models representing different levels of oedema, compared to data classified by the level of oedema $(0=$ no oedema, $4=$ severe oedema). For clarity, the model results are plotted on the same frequency scale as the measured data (frequency range $9.6-614 \mathrm{kHz}$ ). It can be seen that the model correctly predicts a reduction in resistivity across the frequency range with increasing oedema, though the measured data does not show the same pronounced dispersion that the model predicts for normal tissue.

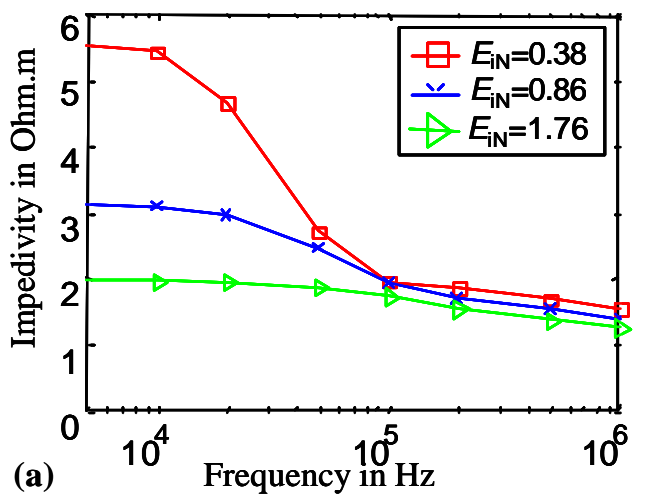

Figure 10

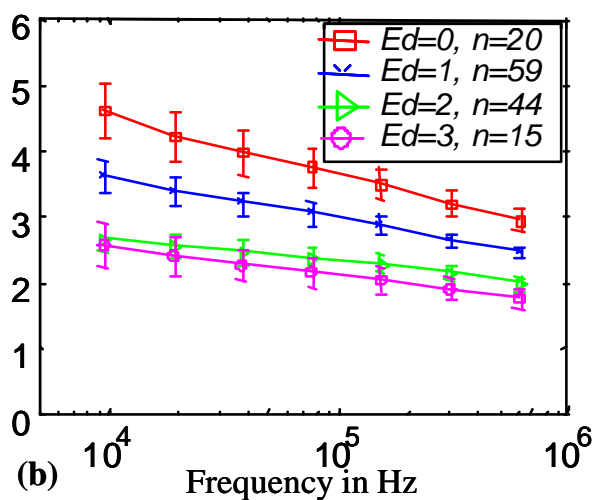

(b) $10 \quad$ Frequency in $\mathrm{Hz}$

(a) Modelled data according to degree of oedema and (b) measured data according to histopathological oedema index $(0=$ no oedema, $3=$ severe oedema). Error bars in (b) represent 1 SEM, and $\boldsymbol{n}$ the number of spectra for each classification.

Figure 11 shows a comparison of the modelled resistivity spectra according to inflammation, and the measured spectra, classified according to the histopathological diagnosis of biopsies taken at the point of data collection. Similarities in the characteristics of the two sets of spectra are apparent, including almost linear appearance over his frequency range, and increase in resistivity at all frequencies as lymphocyte density/inflammation index increases. 

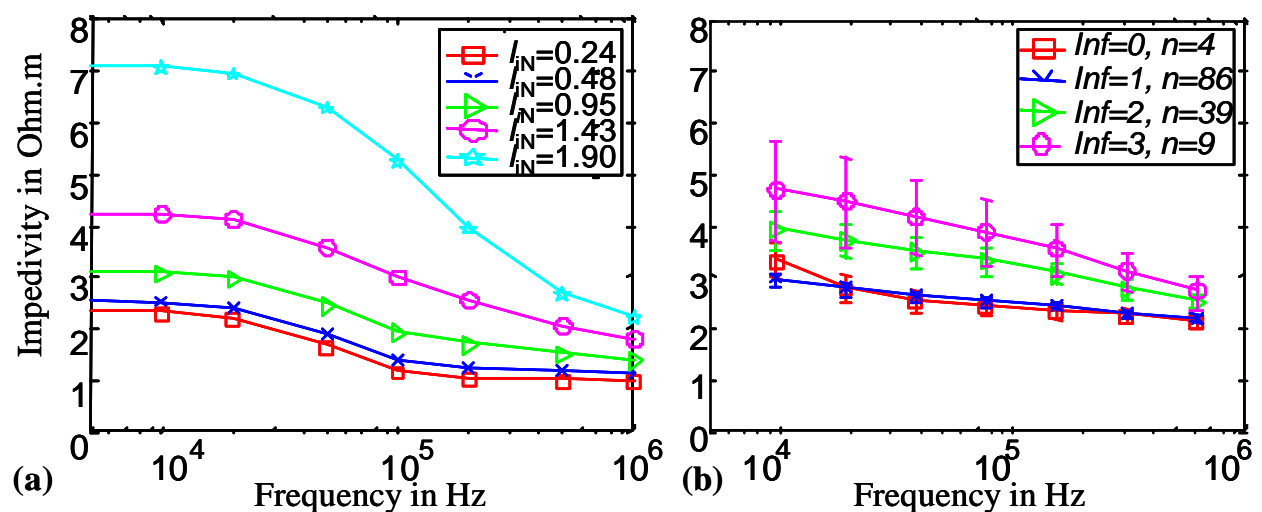

Figure 11

(a) Modelled data according to degree of inflammation and (b) measured data according to histopathological inflammation index $(0=$ no inflammation, $3=$ severe inflammation). Error bars in (b) represent $1 \mathrm{SEM}$ and $\boldsymbol{n}$ the number of spectra for each classification

\section{DISCUSSION}

In contrast to cervical tissue where early stages of malignant progression are confined to the epithelium, loss of barrier function in the urothelium allows urine to penetrate into the underlying tissue, and thus evoke oedema and inflammation. Malignant bladder tissue, unlike several other epithelial tissue types, exhibits an increase in electrical resistivity relative to the undiseased state. Finite Element Modelling has been employed as a tool to investigate which of the features associated with early CIS in the bladder might account for this unusual behaviour.

Modelling the microscopic structure of superficial bladder connective tissue suggests that increasing oedema (in this instance defined as increased capillary leakiness, giving rise to an increase in extracellular conductivity) causes a small reduction in resistivity across all frequencies. By contrast, increasing inflammation, defined as an increase in the density of lymphocytes, was predicted to have a much more significant effect, particularly at low or mid range frequencies.

On a macroscopic tissue scale, the definition of oedema was widened to include increasing extracellular volume in the urothelium - the phenomenon that gives rise to the increase in capillary permeability in the lamina propria. The effects of oedema and inflammation were then quantitatively compared with those of loss of differentiation in the urothelium. In this case increasing oedema was associated with a significant decrease in low frequency 
impedance, a less significant decrease at high frequencies, and an increase in characteristic frequency. Comparison with the lamina propria only model suggests that the low-mid frequency effects are primarily due to the widening of extracellular space, which supports previous sensitivity analyses carried out with single cell models (Walker 2001).

Multivariate statistical analysis on mode lled data suggested that loss of anisotropy would also tend to decrease the low frequency resistivity $R$, whilst an increase in the lymphocyte population in the lamina propria would tend to increase $R$. All three structural changes tend to increase the characteristic frequency. Changes in epithelial cell morphology did not affect high frequency properties, whereas increasing oedema led to a decrease, but inflammation to an increase in $Z_{\text {fmax }}$. Progressive levels of oedema and inflammation appear to have opposite effects on electrical characteristics at both ends of the frequency spectrum, with inflammation increasing, and oedema decreasing the measured impedance.

The relative value of $b$ coefficients yielded by the statistical analysis, suggests that the influence of the three parameters on the low frequency resistivity and characteristic frequency is similar, but inflammation has the most significant effect on high frequency properties.

Comparison with the model results relating to increasing oedema with those classified according to oedema index independently assigned to biopsies by a bladder pathologist, reveals a general agreement in the sense that resistivity across the frequency range tends to decrease with increasing abnormality. However, the model predicts larger changes at low frequency than is shown by the measured data, but underestimates the differences at higher frequencies (above the $10 \mathrm{kHz}$ range). In addition, the modelled spectra exhibit a distinct dispersion, whereas the measured spectra are more linear in form. As our measurement system has been used to collect impedance data from other tissue types, including cervical epithelium which produces more familiar curved spectra with a distinct beta dispersion (Walker D C et al. 2002), it is unlikely that the curve shown in figures 10 and 11 is significantly influenced by measurement artefact.

Due to relatively small sample numbers, the measured spectra were not subdivided according to inflammation or epithelial changes, which could partially account for the discrepancies 
between modelled and measured data. It is also possible that we have overestimated structural changes in the epithelium extracellular volume, which tends to have more influence on the low frequency characteristics, and underestimated changes in the lamina propria, which will have more influence at higher frequencies. As the values of urothelial extracellular volume used in this model were based primarily on knowledge of the ultrastructure of squamous epithelia \{Walker, $2001 \# 102\}$, \{Walker D C, $2003 \# 22\}$, this is certainly a possibility. However, without access to high magnification TEM image of urothelium, it will be difficult to address this problem. Even with access to such images, parameters are likely to be unreliable due to cell swelling or shrinkage associated with the fixation process.

Model parameters relating to the blood vessels were based on the mean size and density of capillaries visible in a small number of histology sections, and parameters published in the literature. A more detailed survey of the histology would be required in order to accurately ascertain the capillary size and density associa ted with various tissue pathologies, and hence obtain a more reliable idea of the contribution of increased capillary permeability to the electrical properties of the lamina propria. In addition, this study focused only on aspects of lamina propria structure that are visible histologically: blood vessels and cells. Collagen fibre density and orientation are also features that might be expected to change with oedema, and until these changes can be quantified, it is impossible to assess their possible effect on electrical impedance

Comparison of the modelled and measured results classified according to inflammation, reveals a similar pattern in changes across the frequency range, at least for lymphocyte densities up to $60 \%$ of the total volume (or $I_{i N}=1.43$ ). As the volume densities modelled were arbitrary, it is possible that densities greater than this are not observed clinically. Spectra appear approximately linear in form across this range, and impedance is increased at all frequencies with increasing inflammation index. These changes can be explained by the fact that cell membranes have a high reactance at low frequencies, thus increasing cellular content in a tissue will effectively reduce the volume available for current flow and hence increase the effective impedance. At high frequencies, a displacement current will flow 
within the cell, but as the conductivity of the cytoplasm is less than that of the surrounding extracellular fluid (see Table 1), the effect of increasing cellular density is still an increase in measured impedance.

This pattern of increasing impedance with progression of inflammation provides a plausible explanation of our previously unexpected observation that unlike cervical tissue, abnormal bladder exhibits an increase in electrical impedance relative to normal tissue: in bladder, the reduction in electrical impedance due to oedema is more than compensated by an increase in impedance caused by lymphocyte invasion of the connective tissue. Although our sensitivity analysis suggests that oedema and inflammation would have approximately equal and opposite effects in the low frequency range, as discussed earlier, it is likely that we have overestimated the change in extracellular volume in the urothelium, which is one of the most difficult parameters to quantify, and hence the reduction in impedance at low frequencies may be significantly smaller than predicted by the model. This hypothesis that inflammation is the dominant effect is supported by the fact that the spectra measured from the small number of samples histologically classified as being both oedema- and inflammation-free $(E d=0, \operatorname{Inf}=0)$ have a significantly smaller impedance across the frequency range than tissue with moderate to high levels of oedema and inflammation ( $E d=3, \operatorname{Inf}=2$, data not shown).

If EIS is to be used clinically for the detection of malignancy in patients undergoing endoscopic bladder examination, the ability to distinguish regions of benign inflammation, from those with malignant potential is essential. The modelling presented here suggests that the effects of connective tissue inflammation may be separated from those resulting from epithelial changes at high frequencies. However, the absolute magnitude of measurements at the high frequency range tend to be smaller, and it may be the case that factors that determine the repeatability of measurements in the data collection system (pressure applied to the probe, surface fluid, inter-patient variability) mean that obtaining reliable values, and hence interpreting tissue structure in this range may be more difficult. Further clinical studies at an extended range of frequencies are already under way, and should go some way to answering this question. Extension of the model to include other features of inflammation and malignant changes, for instance epithelial denudation, will also provide a useful means 
of unravelling the often counter-intuitive effects of changes in tissue structure on the electrical properties of bladder, and also in optimization of the electrode array desig $n$ in order to 'focus' the current flow at a particular depth of interest.

\section{CONCLUSION}

This study is intended to apply finite element modelling as a tool to improve our understanding of how different aspects of tissue structure at various length scales can influence the measured macroscopic physical properties of that tissue - in this case, the electrical impedivity of bladder. The application of interest in this work was the use of electrical impedance spectroscopy in the diagnosis of bladder pathology, and in particular, we sought to address the question of why malignant bladder tissue exhibited an increase in electrical impedivity relative to normal tissue, whilst the opposite behaviour was known to be true for cervical tissue. Whilst this modelling technique has previously been used to examine the electrical characteristics of epithelium, we have now extended the model to include aspects of connective tissue structure, including the properties and morphology of capillaries and lymphocytes.

The modelling results presented here suggest that the inflammatory changes that occur in the connective tissue layer underlying the urinary epithelium, specifically the infiltration of lymphocytes in response to the penetration of urine, may cause an increase in the measured electrical impedivity across the measurement frequency range. This impedivity increase dominates the impedivity reduction that would be expected to be associated with loss of cellular anisotropy and cohesion in the overlying epithelium and oedema in the lamina propria. Further investigation is needed in order to ascertain whether changes in the different tissue layers can be reliably separated, and purely inflammatory changes can be disassociated from the early stages of malignancy.

\section{REFERENCES}


Baluk P, Hirata A, et al. (1997). "Endothelial gaps: time course of formation and closure in inflamed venules of rats." American Journal of Physiology 272(1): L155-70.

Brown, B. H., J. A. Tidy, et al. (2000). "Relation between tissue structure and imposed electrical current flow incervical neoplasia." Lancet 355(9207): 892-5.

Cole, K. S. a. C., R H (1941). "Dispersion and Absorption in Dielectrics.1. Alternating current characteristics." Journal of Chemical Physics 9: 341-351.

Feldman, D., S. L. Romney, et al. (1984). "Ultrastructure of normal, metaplastic and abnormal human uterine cervix:Use of montages to study the topographical relationship of epithelial cells." American Journal of Obstetrics and Gynecology 150(5): 573-688.

Foster, K. R. a. S., H P (1989). "Dielectric properties of tissues and biological materials: a critical review." Critical Reviews in Biomedical Engineering 17: 25-105.

Gabriel, S., R. W. Lau, et al. (1996). "The dielectric properties of biological tissues: III. Parametric models for the dielectric spectrum of tissues." Physics in Medicine and Biology 41: 2271-2293.

Geddes, L. E. and L. A. Baker (1967). "The specific resistance of biological material - a compendium of data for the biomedical engineer and physiologist." Medical and Biological Engineering 5: 271-293.

Gonzalez-Correa, C. A., B. H. Brown, et al. (1999). "Virtual biopsies in Barrett's Esophagus using an impedance probe." Annals of the New York Academy of Sciences 73: 313-322.

Jones D M, Smallwood R H, et al. (2003). "Modelling of epithelial tissue impedance measured using three different designs of probe." Physiological Measurement 24(2): 605623.

Miller C E and H. C. S (1990). "Finite Element Analysis of bioelectric phenomena." Critical Reviews in Biomedical Engineering 18(3): 207-233.

Smallwood R H, Keshtar A, et al. (2002). "Electrical Impedance Spectroscopy (EIS) in the urinary bladder: the effect of inflammation and Edema on identification of malignancy." IEEE Transactions on Medical Imaging 21(6): 708-710.

Walker D C, Brown B H, et al. (2003). "A study of the morphological parameters of cervical squamous epithelium." Physiological Measurement 24: 121-135.

Walker D C, Brown B H, et al. (2002). "Modelled current distribution in cervical squamous tissue." Physiological Measurement 23(1): 159-168.

Walker, D. C. (2001). Modelling the Electrical Properties of Cervical Epithelium.

Department of Medical Physics and Engineering, University of Sheffield, U. K.

White, F. H. and K. Gohari (1984). "Alterations in the Volume of the Intercellular Space

Between EpitheliatCells of the Hamster Cheek-Pouch - Quantitative Studies of Normal and Carcinogen-Treated Tissues." Journal of Oral Pathology \& Medicine 13(3): p.244-254.

Wilkinson B A, Smallwood R H, et al. (2002). "Electrical Impedance Spectroscopy and the diagnosis of bladder pathology: a pilot study." Journal of Urology 168: 1563-1567. 